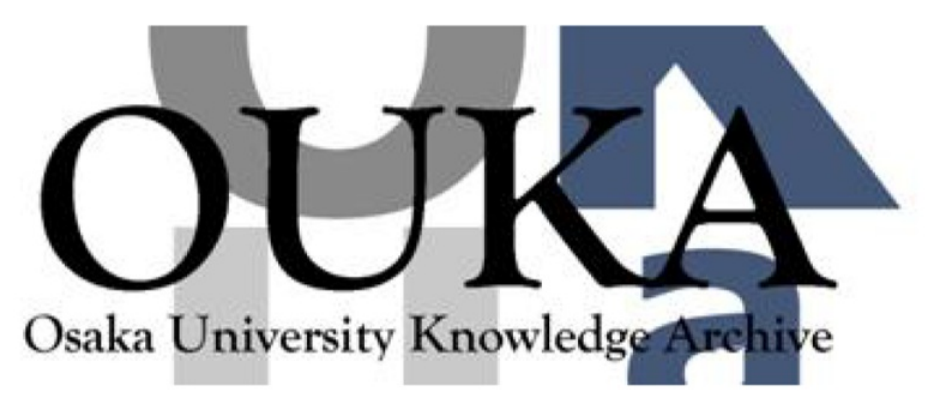

\begin{tabular}{|c|c|}
\hline Title & $\begin{array}{l}\text { High-speed non-contact defect imaging for a } \\
\text { plate-like structure }\end{array}$ \\
\hline Author (s) & Hayashi, Takahiro \\
\hline Citation & NDT and E International. 85 p. 53-p. 62 \\
\hline Issue Date & $2017-01$ \\
\hline oaire:version & AM \\
\hline URL & https://hdl. handle. net/11094/84495 \\
\hline rights & $\begin{array}{l}\text { () } 2016 \text { Elsevier Ltd. This manuscript version is } \\
\text { made avai lable under the Creative Commons } \\
\text { Attribution-NonCommercial-NoDerivatives } 4.0 \\
\text { International License. }\end{array}$ \\
\hline Note & \\
\hline
\end{tabular}

Osaka University Knowledge Archive : OUKA

https://ir. Library. osaka-u. ac. jp/

Osaka University 


\title{
High-speed non-contact defect imaging for a plate-like structure
}

Takahiro Hayashi

Graduate School of Engineering, Kyoto University, Kyoto 615-8540, Japan

\begin{abstract}
Images of defects in a plate-like structure can be obtained from amplitude distributions measured using the scanning laser source technique. This paper discusses high-speed non-contact imaging for defects in a plate. Fundamental experiments conducted using a contact piezoelectric transducer as a receiving device revealed the effect of the generated wave shape and measurement speed on the defect images. Generating tone-burst waves consisting of multiple frequency components and averaging the images obtained in multiple frequency bands (frequency image averaging, FIA) gave clearer defect images in which noisy effects due to low signal-to-noise ratio and spurious images due to multiple reflections in high-speed imaging were reduced. Moreover, FIA was shown to be effective for high-speed non-contact imaging using a laser Doppler vibrometer.
\end{abstract}

Keywords: elastic wave, defect imaging, high speed, scanning laser source, non-contact

Corresponding author: Takahiro Hayashi, Graduate School of Engineering, Kyoto University

C3 Kyotodaigaku-katsura, Nishikyo-ku, Kyoto, Japan

Telephone/fax: +81-75-383-3797 e-mail: hayashi@kuaero.kyoto-u.ac.jp 


\section{Introduction}

Periodic inspections are required to maintain large structures as pipes, bridges, and aircraft appropriately. Strain and temperature measurements using optical and infrared cameras are effective means for rapidly inspecting the surfaces of such structures. However, X-ray and ultrasonic inspections are required to detect internal defects in pipes and delamination in the bodies and wings of aircraft. Ultrasonic inspection is preferred in practical applications because, unlike X-ray inspection, it does not require large equipment for radiation protection.

The most reliable and widely used inspection technique for large structures is the ultrasonic pulse echo technique, which uses longitudinal or transverse elastic waves. However, this technique can evaluate only a small region of the propagation path beneath the ultrasonic transducer in a single measurement; the inspection of large structures is extremely time-consuming and expensive. Therefore, elastic waves propagating along baror plate-like structures, called guided waves, are employed in the inspection of such large structures [1-5].

The authors have investigated effective inspection using guided waves. One study on guided wave inspection focused on the imaging of defects in a pipe using pulse echo signals of guided waves [6], whereby multiple transducers placed along the circumference of the pipe at regular intervals received pulse echo signals from defects, weld lines, pipe ends, and so on. Then, signal processing based on the modal expansion theory of guided waves in a pipe was adopted to create a defect image of the pipe. However, this imaging technique cannot provide appropriate defect images over a wide range (say, over $10 \mathrm{~m}$ ) because of the difference between theoretical and actual velocities in a pipe. This shortcoming limits the practical use of the technique [7]. Therefore, we investigated a new defect imaging technique for plate-like structures, in which elastic waves are generated by 
a pulse laser in a remote area and the source of elastic wave (laser spot) is scanned over a plate-like structure [8-13].

This paper describes the effect of high-speed imaging by the scanning laser source (SLS) technique and investigates high-speed non-contact defect imaging using a laser Doppler vibrometer (LDV) at low signal-to-noise ratio (SNR).

Many publications can be found on laser ultrasonics, in which elastic wave, usually ultrasonic range, is generated by a pulse laser and the vibration is detected with laser interferometry [14-23]. The laser ultrasonics have also been applied to non-destructive evaluation of thin plates for a long time; for example, Hutchins et al. elaborated Lamb wave propagation generated with a laser and showed that the measurements of Lamb waves enable to estimate the plate thickness and their elastic constants [15]. Thereafter, many works were carried out on Lamb waves generated with a laser because an A0 mode of Lamb waves can be generated with relatively large amplitude by laser emission on a plate surface [16-18]. Moreover, non-contact measurements using a laser have also been investigated for imaging material characteristics and defects in a material, in which spatial information was collected by scanning both lasers for generation and detection (or a specimen) [19-21].

In the scanning measurements, elastic wave generation is easily realized through the thermoelastic effect or ablation at the pulsed laser spot, whereas elastic wave detection is strongly affected by the surface condition of the laser spot because laser interferometric wave detection requires scattered or reflected light from the surface. Therefore, wave detection with high-speed scanning is not practical; it requires time-consuming point-by-point measurements and stable light detection in order to measure elastic waves over a wide area with two-dimensional scanning.

In the SLS technique, elastic waves are detected at a fixed position with contact 
ultrasonic transducers or non-contact electromagnetic transducers, air-coupled transducers, and a laser, and only a laser elastic wave source is rastered [24-27]. A large number of signals received for different laser source positions produce spatial information of the material by appropriate signal processing. For example, Kromine et al., Fomitchov et al., and Sohn et al. experimentally and numerically showed that the SLS technique achieves highly sensitive detection of surface-breaking defects [24-26]. Takatsubo et al. developed a visualization technique of wave propagation using signals measured with the SLS technique and adopted this approach for non-destructive evaluation of a material [27].

The authors have studied an imaging method for defects in a plate using the SLS technique. It was shown that the intensity of signal components below the cutoff frequency of the A1 mode of a Lamb wave corresponds to the plate thickness at the laser spot and that defect images can be created from amplitude distributions of the received signals using the SLS technique [8]. Further, the frequency dependence of the defect imaging technique was investigated [9], and non-contact detection was realized using low-frequency air-coupled transducers [11]. Moreover, spurious images were reduced by averaging multiple images obtained by the signals detected at multiple receiving transducers. Fiber laser equipment has been shown to enable the generation of narrowband tone-burst elastic waves and the creation of defect images even with low SNR [12]. In addition, it has been shown that averaging images at different frequencies (frequency image averaging) reduces spurious images because the spurious images caused by multiple reflections and resonances in a specimen have different patterns at different frequencies. Furthermore, defect imaging using the SLS technique has been shown to be effective even for a plate with complex geometries, whereby a direct wave propagating along the straight path from the source to the receiver cannot be detected [12].

This paper discusses high-speed non-contact defect imaging for the purpose of its 
application to the inspection of large structures such as pipes. To establish remote non-contact measurement, laser interferometry is the only solution for receiving vibrations from a remote place. As described previously, wave detection with a laser is strongly affected by the surface condition. However, in defect imaging using the SLS technique, once we select a receiving point where an LDV can measure the vibration stably, we can measure all waveforms for all laser source points because the receiving position does not change.

\section{Principle of defect imaging using the SLS technique}

Before showing experimental results of high-speed imaging, this section describes the principle of the defect imaging for plate-like structures. In the defect imaging technique, the laser source of elastic wave is rastered by galvano mirror scanners and then distributions of an amplitude peak or a Fourier spectrum peak are obtained from the waveforms at all rastering points. Then generation energy of flexural vibration was enhanced when a laser source is located on a defect or in the vicinity of a defect and distributions of the vibration energy correspond to a defect image. The authors analyzed the energy enhancement using semi-analytical finite element calculations and normal mode expansion theory $[8,13]$. Figure 1 shows the schematic figure of the principle: (a) for a plate with a widely spread defect like erosion and corrosion and (b) for a plate with a non-volume crack stretching in the thickness direction. In (a), when the laser beam is irradiated on an intact thick area, small flexural vibration is generated, whereas, if the laser source is located at a thin defected area, the detected signals at the LDV become large. It is shown in ref. [8] that this phenomenon appears at the frequency range below the cut-off frequency of A1 mode. In (b), if laser beam is irradiated in the vicinity of a reflective object like a crack, the interaction of a pair of evanescent waves from the laser source and 
the reflection object generates extra vibration energy, whereas if the laser source locates far from the reflection object, the interaction of the evanescent waves becomes small and the energy enhancement cannot be seen. The energy enhancement caused by the interaction of evanescent modes was discussed in ref. [13].

\section{Experimental system and specimen used}

Fundamental experiments on high-speed imaging were conducted under high SNR using contact piezoelectric transducers before high-speed non-contact imaging was examined using an LDV as a receiving device.

Figure 2 shows the experimental setup used in this study. A fiber laser with a maximum output of $50 \mathrm{~W}$ (SPI laser, G4-HS) was used for generating elastic waves. The laser equipment could emit pulse trains with a high repetition rate of over $100 \mathrm{kHz}$ and could control the laser output by external modulation signals. In this study, pulse trains at a repetition rate of $540 \mathrm{kHz}$ were modulated by the external signals shown in Fig. 3, where the laser would output and stop at high and low levels of the modulation signal, respectively; consequently, intermittent loading and unloading were repeated at the laser spot. The generation of narrowband burst waves with laser pulse trains and modulated laser output can be referred to [12], [22], and [23].

As shown in Fig. 3 (a), one square wave for one trigger TTL from the galvano mirror controller was used for generating a pulse wave. Multi-cycle square waves, shown in Fig. 3 (b), were used for generating narrowband tone-burst waves. Figure 3 (c) shows a modulation waveform formed by the connection of square waves of three different frequencies. This will be used in Section 5.2 to generate narrowband tone-burst waves having three frequency components. Figures 3 (d)-(f) are frequency spectra for the modulation signals shown in Figs. 3 (a)-(c), respectively. These frequency spectra show 
that one square wave (a) and multi-cycle square burst waves consist of broadband and narrowband frequency components, respectively, and (c) contains three distinct peaks at the desired frequencies $(7,9$, and $11 \mathrm{kHz})$, together with DC components and 3rd and 6th harmonic components. The external modulation signals were generated by the field-programmable gate array (FPGA) module (National Instruments NI FlexRIO) at the trigger TTL from the galvano mirror controller. At the trigger timing, the galvano mirror moved to the next rastering point repeatedly, i.e., the scanning speed increased as the trigger TTL was repeated at a higher repetition rate.

Figure 4 is light intensities scattered at the surface of a specimen and detected by an amplified photo detector. Figures 4 (a)-(c) correspond to the laser intensities for the modulation signals shown in Figs. 3 (a)-(c), and Figs 4 (d)-(f) are their Fourier spectra. The light intensities and the Fourier spectra agree well with the modulation signals as shown in Fig. 3, which proves that the laser pulse trains were irradiated following the modulation signals. The zoomed figure of (a) in the inset of Fig. 4 show that the pulse trains are comprised of small laser pulses at the repetition frequency of $540 \mathrm{kHz}$.

The elastic waves generated by the laser emission propagated in an aluminum alloy plate, as shown in Fig. 5, and were received on the surface of the right edge. Two receiving devices were employed: one was a contact piezoelectric transducer for fundamental experiments with high SNR, and the other was an LDV (Polytec OFV-5000) for non-contact imaging. The received signals were amplified to $60 \mathrm{~dB}$ and then digitized at a sampling frequency of $200 \mathrm{kHz}$ with a 12-bit AD converter (National Instruments NI 5752). Band-pass filtering between $2 \mathrm{kHz}$ and $13 \mathrm{kHz}$, fast Fourier transform (FFT), and image processing were implemented using a personal computer, and the defect images could be obtained at nearly the same time as the signal measurements.

Figure 5 shows the dimensions of the aluminum alloy plate used and the positions of the 
laser emission region and receiving points. A thru notch was sawed at the center of the aluminum alloy plate (A5052) measuring $500 \mathrm{~mm} \times 500 \mathrm{~mm} \times 3 \mathrm{~mm}$. Laser pulses were emitted for elastic wave generation in the left region measuring $80 \mathrm{~mm} \times 80 \mathrm{~mm}$. The thru notch disturbed the straight path between the laser sources and the receiving points, and the received signals consisted of only waves passing through detour paths by diffraction and reflection. It has been shown that defect images can be obtained even for a plate with complex geometries using the same plate specimen [12]. An artificial defect, i.e., the letter "K", was engraved to a depth of $1.5 \mathrm{~mm}$ behind the laser emission region. A piezoelectric transducer was attached to the plate surface at $50 \mathrm{~mm}$ above the right bottom corner, and a receiving point with an LDV was set at $120 \mathrm{~mm}$ from the right bottom corner, where a retro-reflective marker was attached to improve the detectability of the scattered laser beam from the surface.

\section{High-speed defect imaging with broadband pulse wave}

\subsection{Comparisons of defect images, waveforms, and frequency spectra at different repetition rates}

First, high-speed imaging was examined using the modulation signal for pulse wave generation, as shown in Fig. 3 (a). Figures 6 (a) and (b) show examples of defect images at low speed, where the repetition rate of the trigger TTL in the experimental system described above was set to $20 \mathrm{~Hz}$. On the other hand, Figures 7 (a) and (b) show defect images in high-speed measurements at a repetition rate of $200 \mathrm{~Hz}$. The galvano mirrors moved to the next rastering point at the trigger timing and the laser source was scanned over the region. Here, the laser source was rastered along one line from left to right at 1-mm increments and then moved up to the next line at 1-mm increments. Repeating the scanning over the $80 \mathrm{~mm} \times 80 \mathrm{~mm}$ region yielded two-dimensional distributions of the 
signals. Consequently, laser emission and mirror rotation were repeated every $1 / 20 \mathrm{~Hz}=50$ ms and $1 / 200 \mathrm{~Hz}=5 \mathrm{~ms}$ for $81 \times 81$ points and it took around $328 \mathrm{~s}$ and $33 \mathrm{~s}$, respectively, to measure the signals. It should be noted that the laser spot seemed to move continuously in one line because the laser scanning was sufficiently fast. Therefore, the laser spot might suffer from small position errors.

Figure 6 (c) shows the waveform detected when the laser was emitted at the center of the laser emission region at a repetition rate of $20 \mathrm{~Hz}$. The figure shows a waveform for an interval of $10 \mathrm{~ms}$ after the AD converter received the trigger TTL. Although the signals are not clear owing to low SNR, a received waveform seems to appear after around $1 \mathrm{~ms}$. The frequency spectrum of the waveform for $10 \mathrm{~ms}$ is shown in Fig. 6 (d). This spectrum is normalized by the peak value between $8 \mathrm{kHz}$ and $10 \mathrm{kHz}$, and then, the frequency spectra are also normalized by the same value for comparison of intensity. The large peak at 15 $\mathrm{kHz}$ is the intrinsic noise of the pre-amplifier; the noise occupies the major part of the waveform in Fig. 6 (c). Figure 6 (a) shows the distribution of the maximum value of the radio frequency (RF) waveform for $10 \mathrm{~ms}$ (RF image), and Fig. 6 (b) shows the distribution of the peak value of the frequency spectrum between $8 \mathrm{kHz}$ and $10 \mathrm{kHz}$ (FFT image). In both figures, the intensity is shown with a gray scale in which black and white represent the maximum and minimum values, respectively. In Fig. 6 (a), we can see a non-smooth sandstorm distribution because random noise in the waveforms has a strong effect owing to low SNR. Meanwhile, in Fig. 6 (b), the sandstorm effect is reduced but the number of spurious images around the defect image increases. This is the effect of multiple reflections contained in the waveforms for the interval of $10 \mathrm{~ms}$. Multiple reflections have been discussed in detail in previous studies [11, 12].

Figures 7 (a) and (b) show RF and FFT images for a repetition rate of $200 \mathrm{~Hz}$. These images were also obtained from the maximum values of the RF signals and frequency 
spectrum peaks between $8 \mathrm{kHz}$ and $10 \mathrm{kHz}$, as in the case of Figs. 6 (a) and (b). However, at the high repetition rate, the waveforms were not recorded at every laser emission spot; recording started at the left edge and one waveform was recorded continuously until the right edge. Then, we extracted waveforms corresponding to all the laser spots and constructed the images using the extracted waveforms. Figure 7 (c) shows the extracted waveforms for the interval of $10 \mathrm{~ms}$ from the moment when the laser was located at the center of the laser emission region. Because the laser emission was repeated every $5 \mathrm{~ms}$ $(=1 / 200 \mathrm{~Hz})$ at slightly different positions, the current extracted waveform also contains waveforms generated by the next laser emission. A comparison of the waveforms in Fig. 6 (c) and Fig. 7 (c) shows that the signal level slightly increases in Fig. 7 (c) because multiple reflected waves and waves generated by the previous laser shot were superposed at the high repetition rate. Consequently, the sandstorm effect caused by low SNR was reduced, as seen by comparing Fig. 7 (a) with Fig. 6 (a). Meanwhile, spurious images increased, as seen by comparing Fig. 7 (b) with Fig. 6 (b), because at the high repetition rate, the image was more strongly affected by the resonances of multiple reflections and high-repetition wave generation.

\subsection{Effect of time interval of extracted waves}

In Figs. 7 (a) and (b), all the signals from the left edge to the right edge were recorded at one time, and then, the images were obtained from waveforms extracted for the time interval of $10 \mathrm{~ms}$. Because the extracted waveforms varied with multiple reflections and repetitions of laser emission, we could infer that the interval of the extracted waveform affects the images. To evaluate the effect of the interval, Fig. 8 shows RF and FFT images for intervals of $1 \mathrm{~ms}$ and $30 \mathrm{~ms}$, where the repetition rate is set to $200 \mathrm{~Hz}$. As seen in the waveform of Fig. 6 (c), propagation from the laser emission spots to the transducer took 
around $1 \mathrm{~ms}$. Therefore, for a short extraction time interval of $1 \mathrm{~ms}$, the images shown in Figs. 8 (a) and (c) were constructed using waveforms generated in the previous laser emissions. The RF image in Fig. 8 (a) shows the sandstorm effect owing to low SNR, but the artificial defect is obtained appropriately. In the FFT image shown in Fig. 8 (c), the sandstorm effect is reduced, but spurious images appear owing to multiple reflections. For the time interval of $30 \mathrm{~ms}$, the extracted waveforms contained waves generated by six laser emissions at six laser spots. Because the maximum value among the waves at the six laser spots is plotted in Fig. 8 (b), the defect image appears stretched in the scanning direction. The FFT image shown in Fig. 8 (d) contains significant spurious regions because the waveforms for 30 ms consist of a large number of multiple reflections.

In summary, the short interval of the extracted wave causes the sandstorm effect in an RF image owing to low SNR, and the long interval causes deformation of defect images in an RF image as well as spurious images in an FFT image owing to multiple reflections and multiple laser emissions. Hereafter, in high-speed imaging at a repetition rate of over 200 $\mathrm{Hz}$, the interval of the extracted waveform was set to twice the repetition period.

\subsection{Reduction of spurious images with frequency image averaging}

Spurious images are caused by multiple reflections and high-repetition generation of elastic waves. When considering harmonic wave, spurious images are regarded as resonance patterns in a plate-like structure. Our previous study $[9,11]$ focused on the fact that resonance patterns vary with the receiving positions, and proved that spurious images can be reduced by taking the average of images obtained at different receiving positions. In ref. [12], using the characteristic that the resonance patterns vary with frequencies, spurious images were reduced by taking average of the images at different frequencies. This study also adopts the frequency image averaging (FIA). 
Although we used a pulse wave in this case, unlike the approach followed in [12], FIA was applied to reduce spurious images by taking images in three frequency bands $(6-8 \mathrm{kHz}$, 8-10 kHz, and 10-12 kHz) in which the frequency components were distributed as shown in Fig. 7 (d). Figures 9 (a), (b), and (c) show the distributions of the maximum values of the frequency spectra at the frequency bands, and Fig. 9 (d) shows their average. The repetition rate and the interval of the extracted waves were $200 \mathrm{~Hz}$ and $10 \mathrm{~ms}$, respectively, as in Fig. 7. Spurious images were appropriately reduced. Figure 9 (d) shows the defect more clearly than Figs. 7 (a) and (b). This proves that FIA works well for broadband pulse waves.

\subsection{Defect images in high-speed measurements}

Imaging speed can be increased by employing a higher repetition rate. This subsection discusses the effect of high-speed measurements on defect images. Figures 10 (a)-(c) show defect images using FIA at repetition rates of $600 \mathrm{~Hz}, 1000 \mathrm{~Hz}$, and $2000 \mathrm{~Hz}$ (repetition periods of $1.67 \mathrm{~ms}, 1 \mathrm{~ms}$, and $0.5 \mathrm{~ms}$ ), respectively. As in Fig. 9, three images at three frequency bands were averaged. As described in Section 4.2, the waves were recorded at one line from left to right continuously, and the waves at each laser spot were extracted for the double interval of the repetition period. The required times to obtain the images were around 11, 7, and $4 \mathrm{~s}$, respectively. As the imaging speed increased, the images of the artificial defect, i.e., "K", were more distorted, mainly because of multiple reflections at the high repetition rate. The longitudinal patterns were caused by the jump from the right end to the left edge during laser scanning. Waveforms for laser sources near the left edge consisted of multiple reflected waves generated near the right edge before laser beam jumping. Therefore, significant changes in multiple reflected waves cause such longitudinal noise in the images. 


\section{High-speed defect imaging with narrowband tone-burst wave}

\subsection{Comparisons of defect images, waveforms, and frequency spectra at different repetition rates}

Because the use of narrowband tone-burst waves improves SNR in the frequency domain, it is expected that defect images can be obtained even in a noisy environment [12]. Here, high-speed imaging was examined using square tone-burst modulation signals, as shown in Fig. 3 (b).

Figures 11 (a) and (b) show RF and FFT images obtained from 10-ms waveforms at the low repetition rate of $20 \mathrm{~Hz}$ using the modulation signals; the frequency was $9 \mathrm{kHz}$, duration time was $1000 \mu \mathrm{s}$, and duty ratio was 50\%, as shown in Fig. 3 (b). The images obtained for the tone-burst waves correspond to Figs. 6 (a) and (b) for the pulse waves. Figure 11 (c) shows the waveform when the laser was emitted at the center of the scanning region, and Fig. 11 (d) shows its frequency spectrum. The intensity of the frequency components can be compared with Fig. 6 (d) and Fig. 7 (d) because the frequency spectrum is normalized by the same value. Thus, the frequency spectrum clearly shows that the use of tone-burst wave significantly improves the SNR in the frequency domain. Moreover, wave packets appear after around $1 \mathrm{~ms}$ in Fig. 11 (c), which is the minimum travelling time from the laser spot to the receiving transducer. Because the SNR was improved in both the time domain and the frequency domain, the sandstorm effect in Figs. 11 (a) and (b) was reduced, as compared with Figs. 6 (a) and (b). Spurious regions can be seen even in the RF image because the long duration of the elastic wave causes multiple reflections to a greater extent.

Figures 12 (a) and (b) show defect images at the high repetition rate of $200 \mathrm{~Hz}$. Figure 12 (c) shows the waveform when the laser was emitted at the center of the scanning region, 
and Fig. 12 (d) shows its frequency spectrum. The waveform shown in Fig. 12 (c) seems to repeat at the repetition rate of $5 \mathrm{~ms}$ because the received signals are superposed by the waves generated in the previous laser shots owing to the high repetition rate. Spurious regions slightly increase in Figs. 12 (a) and (b) as compared with Figs. 11 (a) and (b), which indicates that the images at high scanning speed are affected by multiple reflections as well as waves generated at the previous laser shots.

\subsection{FIA using tone-burst waves with multiple frequency components}

Here, defect imaging with FIA was examined for tone-burst wave generation. To generate tone-burst elastic waves with multiple frequency components, we used the modulation signals shown in Fig. 3 (c), in which three square waves with different frequencies are connected. Figures 13 (a)-(d) show distributions from the frequency spectra when the modulation signal is set to square tone-burst waves at frequencies of 7,9 , and $11 \mathrm{kHz}$ with a duration of around $1 \mathrm{~ms}$ each. The distributions (a), (b), and (c) were obtained from the maximum value of the frequency spectrum in the frequency bands of 6$8 \mathrm{kHz}, 8-10 \mathrm{kHz}$, and 10-12 kHz, respectively. Further, Fig. 13 (d) shows the average of Figs. 13 (a), (b), and (c). Figure 13 (e) shows the waveform at the center of the region and Fig. 13 (f) shows its frequency spectrum. The frequency spectrum shown in Fig. 13 (f) is significantly large as compared to the frequency spectrum for pulse generation shown in Fig. 7 (d). Moreover, it has frequency components not only in the frequency band of 8-10 $\mathrm{kHz}$ but also in the frequency bands of $6-8 \mathrm{kHz}$ and $10-12 \mathrm{kHz}$, as compared with Fig. 12 (d), which shows the generation of a tone-burst wave with a single frequency component. Therefore, the sandstorm effect was reduced in these images, as compared with Fig. 9. In addition, Fig. 12 (b), which shows an image for a single-frequency tone-burst wave generation, is nearly the same as Fig. 13 (b). This indicates that an image separated in a 
specific frequency band can be obtained appropriately by taking frequency spectrum peaks in the frequency band. In the average image, i.e., Fig. 13 (d), spurious regions were reduced as compared with Fig. 12 (b), which shows that FIA using tone-burst waves with multiple frequency components works effectively.

\subsection{Defect images in high-speed measurements}

As in the case of pulse wave generation, defect images can be obtained by increasing the repetition rate of laser emission even in the case of tone-burst wave generation. However, when using tone-burst waves, it is necessary to check that the duration of the modulation signal is within the repetition period. For example, when the repetition rate of $600 \mathrm{~Hz}$ (repetition period of $1.67 \mathrm{~ms}$ ) is used and the modulation signal contains three frequency components as shown in Fig. 3 (c), the total duration of the square waves must be less than $1.67 \mathrm{~ms}$. Now, the duration of each square wave is set to $1 / 3$ of the repetition period, i.e., the duration of each frequency component is around $0.55 \mathrm{~ms}, 0.33 \mathrm{~ms}$, and $0.16 \mathrm{~ms}$ for repetition frequencies of $600 \mathrm{~Hz}, 1000 \mathrm{~Hz}$, and $2000 \mathrm{~Hz}$, respectively. Figure 14 shows the average images of the three frequency images for the repetition frequencies of $600 \mathrm{~Hz}, 1000 \mathrm{~Hz}$, and $2000 \mathrm{~Hz}$, which can be compared with Fig. 10 for pulse wave generation. At $600 \mathrm{~Hz}$, the sandstorm effect was reduced because the use of narrowband tone-burst wave improved the SNR. However, for $1000 \mathrm{~Hz}$ and $2000 \mathrm{~Hz}$, the images obtained by pulse wave generation (Figs. 10 (b) and (c)) and tone-burst wave generation (Figs. 13 (b) and (c)) were nearly the same, because the tone-burst durations at the high repetition rate had to be short (less than a few cycles) and the images showed similar tendency to the case of one-cycle pulse generation.

\section{Comparison of images at low SNR}


In the experiments described, contact piezoelectric transducers were used as receiving devices in order to obtain fundamental data at high SNR. If the contact transducer can be replaced by a non-contact laser device, the imaging technique becomes feasible for remote measurements and can be widely applied in practical inspection. In this section, defect imaging with multi-frequency tone-burst FIA is investigated using an LDV as a receiving device.

An LDV was used as a receiving device instead of a piezoelectric transducer, as shown in Fig. 2, and elastic waves were detected at the position shown in Fig. 5. Defect images with multi-frequency tone-burst FIA are shown in Figs. 15 and 16. Because the frequency components between $6 \mathrm{kHz}$ and $8 \mathrm{kHz}$ could not be measured with the LDV, the modulation tone-burst square waves at frequencies of $9 \mathrm{kHz}, 11 \mathrm{kHz}$, and $13 \mathrm{kHz}$ were adopted, and images at different frequencies were obtained by taking the maximum values in the corresponding frequency bands. Figures 15 and 16 show defect images with FIA using pulse wave and tone-burst waves, respectively, with three frequency components. The images obtained by pulse waves (Fig. 15) exhibited the sandstorm effect owing to low SNR, whereas the images obtained by tone-burst waves (Fig. 16) gave apparently clearer defect images. These results show the effect of multi-frequency tone-burst FIA more obviously than the case of Fig. 14. In addition, they show that non-contact measurement with LDV is feasible for defect imaging at high speeds.

\section{Conclusions}

Defect imaging using the scanning laser source (SLS) technique was examined in high-speed measurements. Fundamental experiments using contact piezoelectric transducers were conducted to evaluate defect images in high-speed measurements for broadband pulse generation and narrowband tone-burst generation. For both wave types, 
spurious images were reduced by taking the average of multiple images in different frequency bands (frequency image averaging, FIA). In particular, in narrowband tone-burst generation, clearer images were obtained owing to the improved signal-to-noise ratio (SNR) in the frequency domain. However, in high-speed measurements, such improvement in SNR was not observed in the defect images because tone-burst waves with long duration cannot be generated at a high repetition rate. In the experiments conducted at low SNR using a non-contact laser Doppler vibrometer (LDV) as a receiving device, it was found that FIA with narrowband tone-burst waves is highly effective for defect imaging at high speeds.

\section{Acknowledgement}

This work was supported by JSPS KAKENHI Grant Number 26282094 and Chubu Electric Power Co., Inc. 


\section{References}

[1] J.L. Rose, Ultrasonic waves in solid media, Cambridge University Press, 1999.

[2] D.N. Alleyne, B. Pavlakovic, M.J.S. Lowe, P. Cawley, Rapid long-range inspection of chemical plant pipework using guided waves, Insight 43 (2001) 93-96, 101. [3] P.J. Mudge, Field application of the Teletest (R) long-range ultrasonic testing technique, Insight 43 (2001) 74-77.

[4] P. Cawley, M.J.S. Lowe, D.N. Alleyne, B. Pavlakovic, P. Wilcox, Practical long range guided wave testing: Applications to pipes and rails, Mater. Eval. 61 (2003) 66-74.

[5] J.L. Rose, A baseline and vision of ultrasonic guided wave inspection potential, J. Press. Vessel Technol. 124 (2002) 273-282.

[6] T. Hayashi, M. Murase, Defect imaging with guided waves in a pipe, J. Acoust. Soc. Am. 117 (2005) 2134-2140.

[7] T. Hayashi, M. Nagao, M. Murase, Defect imaging with guided waves in a long pipe, J. Solid Mech. Mater. Eng. 2 (2008) 888-899.

[8] T. Hayashi, M. Murase, M.N. Salim, Rapid thickness measurements using guided waves from a scanning laser source, J. Acoust. Soc. Am. 126 (2009) 1101-1106.

[9] T. Hayashi, M. Murase, T. Kitayama, Frequency dependence of images in scanning laser source technique for a plate, Ultrasonics 52 (2012) 636-42.

[10] M.N. Salim, T. Hayashi, M. Murase, T. Ito, S. Kamiya, Fast remaining thickness measurement using a laser source scanning technique, Mater. Trans. (2012) 1-7.

[11] T. Hayashi, M. Murase, N. Ogura, T. Kitayama, Imaging defects in a plate with full non-contact scanning laser source technique, Mater. Trans. 55 (2014) 1045-1050.

[12] T. Hayashi, Imaging defects in a plate with complex geometries, Applied Physics Letters, 108 (2016) 081901

[13] T. Hayashi, M. Fukuyama, Vibration energy analysis of a plate for defect imaging 
with a scanning laser source technique, J. Acoust. Soc. Am. Under review.

[14] C.B. Scruby, L.E. Drain, Laser ultrasonics: techniques and applications, Adam Hilger, New York, 1990.

[15] D.A. Hutchins, K. Lundgren, S.B. Palmer, A laser study of transient Lamb waves in thin materials, J. Acoust. Soc. Am. 85 (1988) 1441-1448.

[16] J.B. Spicer, a. D.W. McKie, J.W. Wagner, Quantitative theory for laser ultrasonic waves in a thin plate, Appl. Phys. Lett. 57 (1990) 1882-1884.

[17] M.A. Johnson, Y.H. Berthelot, P.H. Brodeur, L.A. Jacobs, Investigation of laser generation of Lamb waves in copy paper, Ultrasonics. 34 (1996) 703-710.

[18] S.E. Burrows, B. Dutton, S. Dixon, Laser generation of lamb waves for defect detection: Experimental methods and finite element modeling, IEEE Trans. Ultrason. Ferroelectr. Freq. Control. 59 (2012) 82-89.

[19] R.J. Dewhurst, Q. Shan, Through-Transmission Ultrasonic-Imaging of Subsurface Defects Using Noncontact Laser Techniques, Opt Laser Eng. 16 (1992) 163-178.

[20] M. Clark, S.D. Sharples, M.G. Somekh, Fast, all-optical rayleigh wave microscope: imaging on isotropie and anisotropie materials, IEEE Trans. Ultrason. Ferroelectr. Freq. Control. 47 (2000) 65-74.

[21] S.D. Sharples, M. Clark, R.J. Smith, R.J. Ellwood, W. Li, M.G. Somekh, Laser ultrasonic microscopy, Nondestruct. Test. Eval. 26 (2011) 367-384.

[22] J.W. Wagner, J.B. Deaton, J.B. Spicer, Generation of ultrasound by repetitively Q-switching a pulsed Nd:YAG laser., Appl. Opt. 27 (1988) 4696-700.

[23] R. Pierce, C. Ume, J. Jarzynski, G.W. Woodruff, Temporal modulation of a laser source for the generation of ultrasonic waves, 33 (1995) 133-137.

[24] A. Kromine, P. Fomitchov, Sridhar Krishnaswamy, J.D. Achenbach, Laser ultrasonic detection of surface breaking discontinuities: scanning laser source technique, 
Mater. Eval. 58 (2) (2000) 173-177.

[25] P.A. Fomitchov, A.K. Kromine, Y. Sohn, S. Krishnaswamy, J.D. Achenbach, Ultrasonic imaging of small surface-breaking defects using scanning laser source technique, in: D. Thompson, D. Chimenti (Eds.), Review of Progress in Quantitative Nondestructive Evaluation, American Institute of Physics, 2002, 356-362.

[26] Y. Sohn, S. Krishnaswamy, Interaction of a scanning laser-generated ultrasonic line source with a surface-breaking flaw, J. Acoust. Soc. Am. 115 (1) (2004) 172-181.

[27] J. Takatsubo, B. Wang, H. Tsuda, N. Tooyama, Generation laser scanning method for the visualization of ultrasounds propagating on a 3-D object with an arbitrary shape, J. Solid. Mech. Mater. Eng. 1 (12) (2007) 1405-1411. 
Fig. 1 Principle of defect imaging using the SLS technique

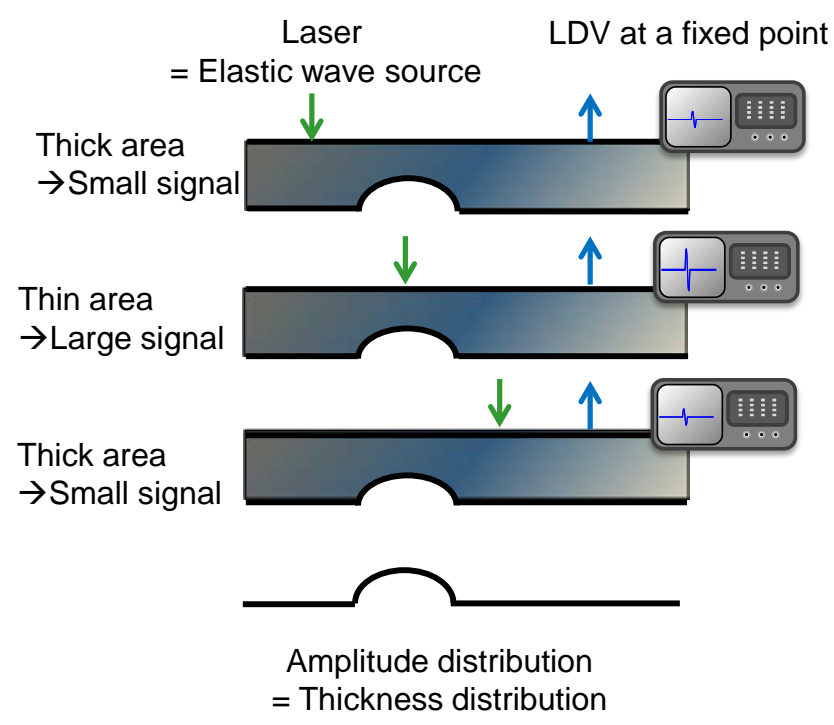

(a) A widely spread defect

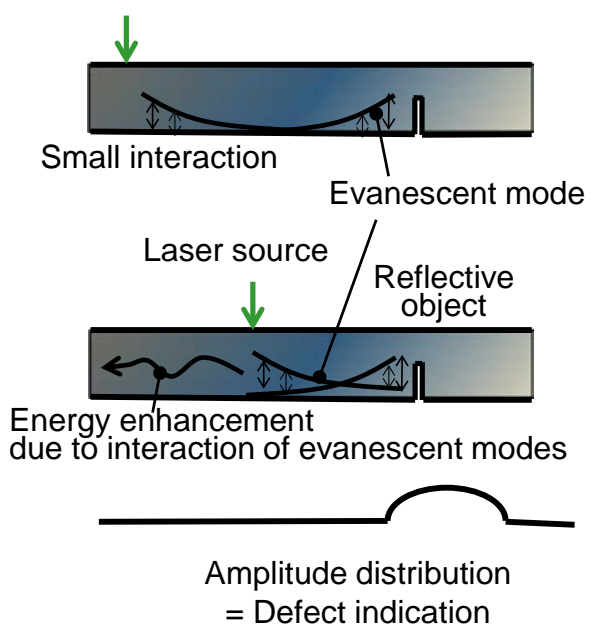

(b) A non-volume crack 
Fig. 2 Experimental setup

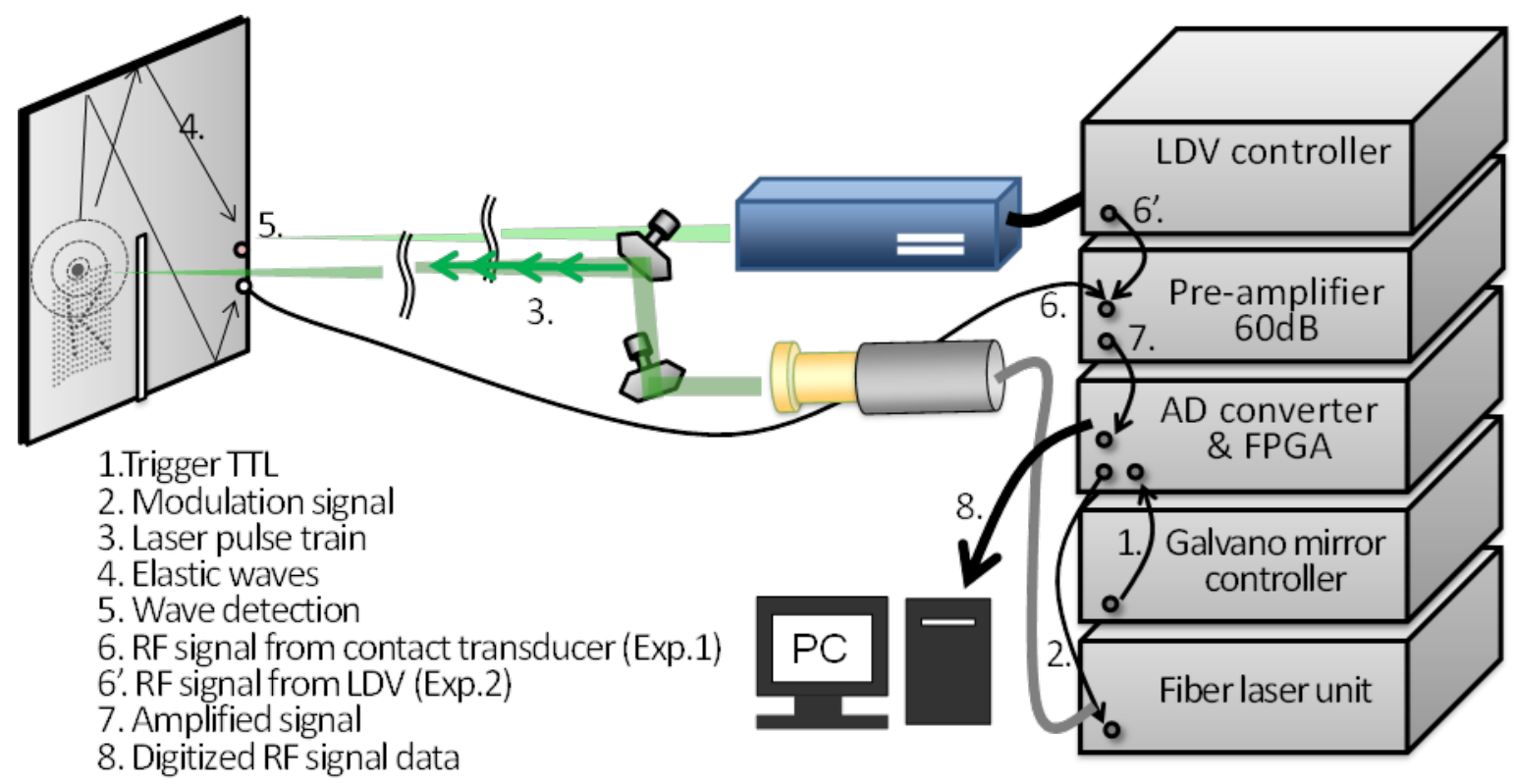


Fig. 3 Typical modulation signals and their Fourier spectra for generation of (a)(d) broadband pulse waves, (b)(e) narrowband tone-burst waves, and (c)(f) multi-frequency narrowband tone-burst waves.

(a)

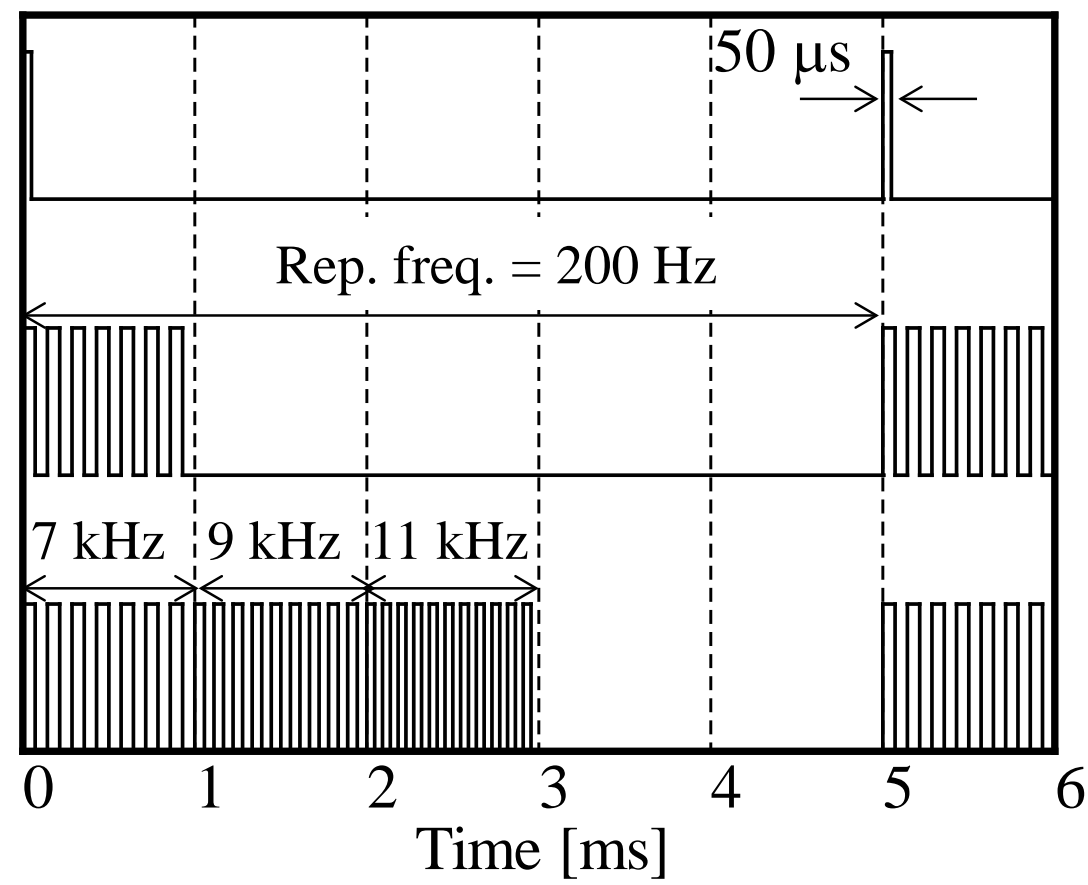

(d)

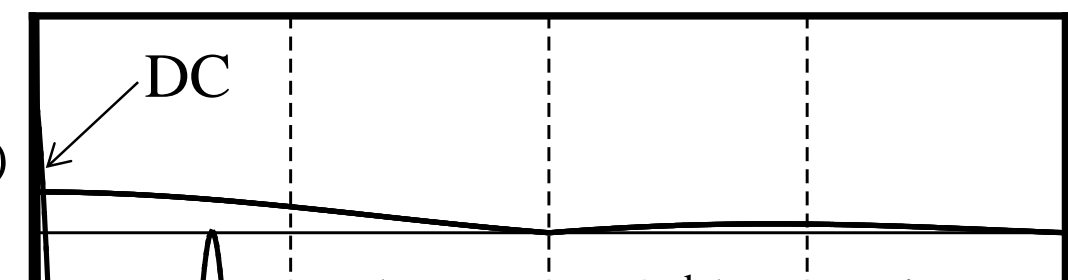

(e)
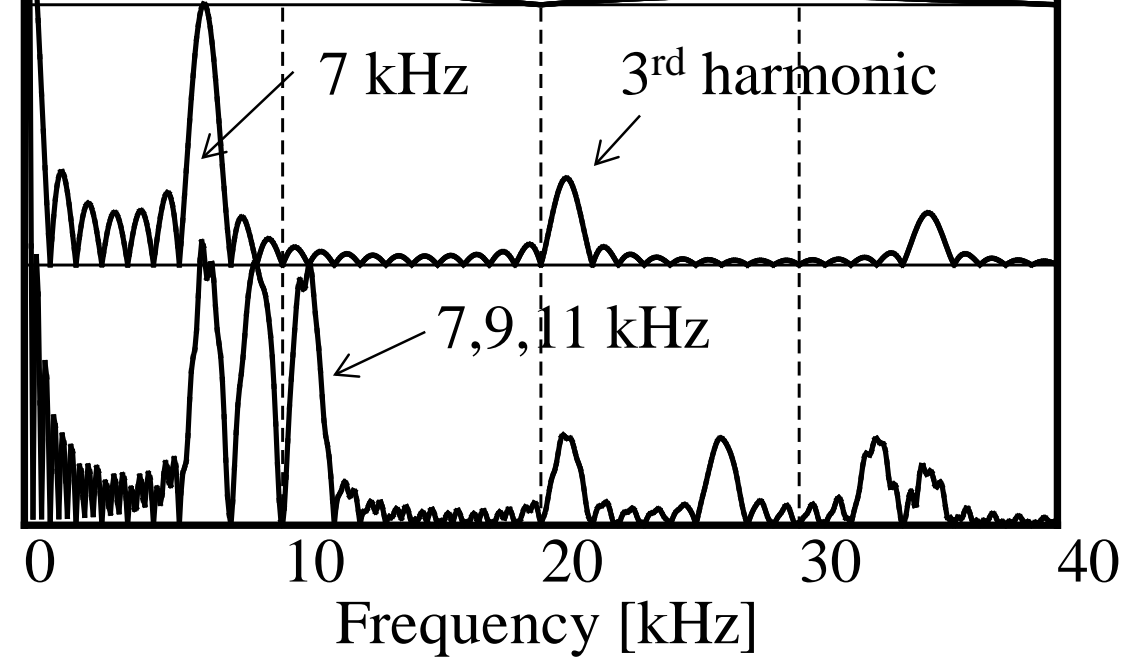

(f) 
Fig. 4 Light intensities detected by a photo detector and their frequency spectra. (a) - (c) are intensities for the modulation signals shown in Figs. 3 (a) - (c), and (d) - (f) are the frequency spectra of Figs. 4 (a) - (c), respectively.

(a)

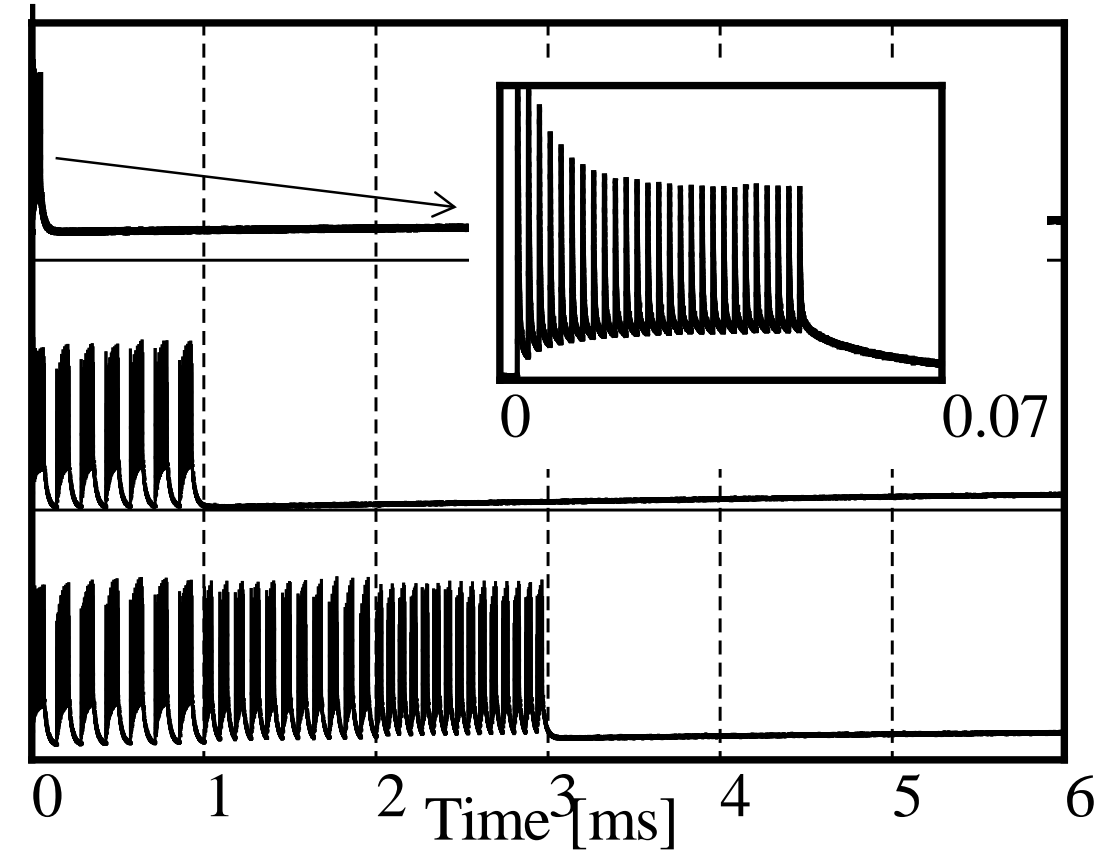

(d)

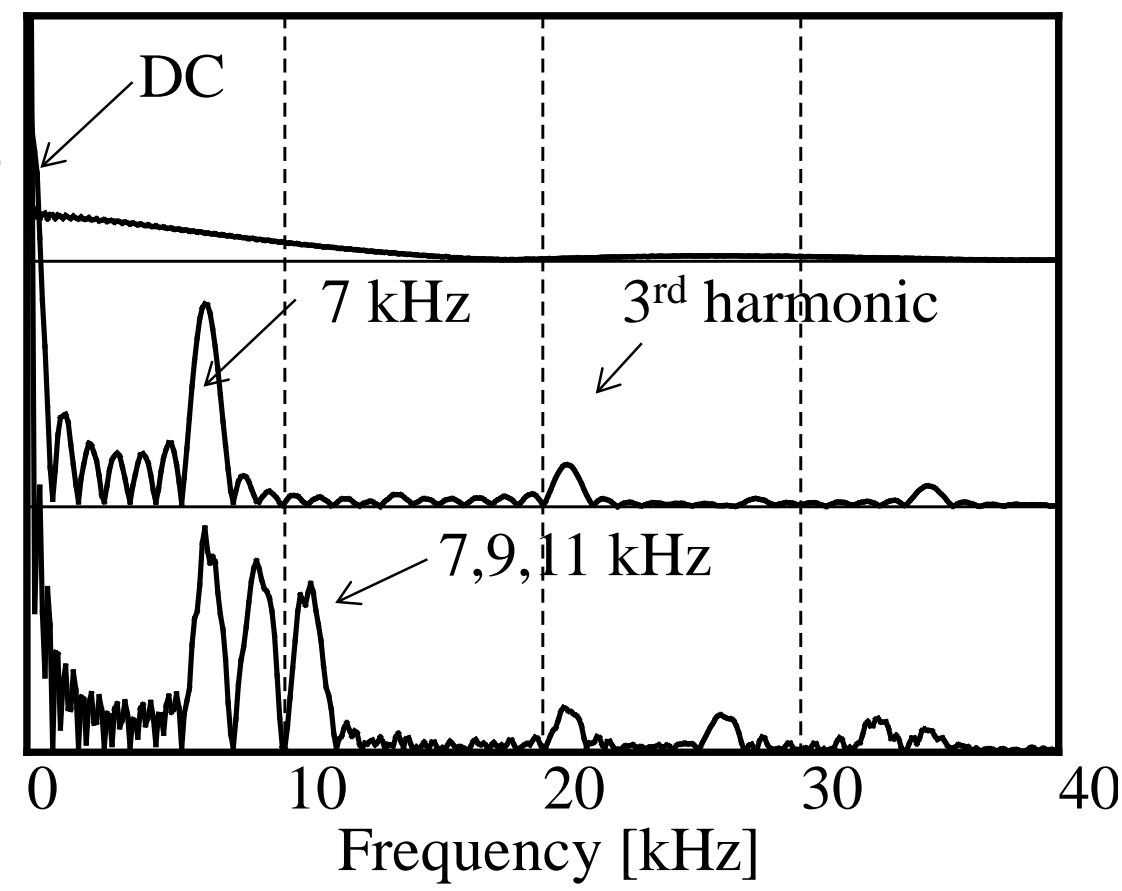


Fig. 5 Specimen used in this study.

Unit: mm

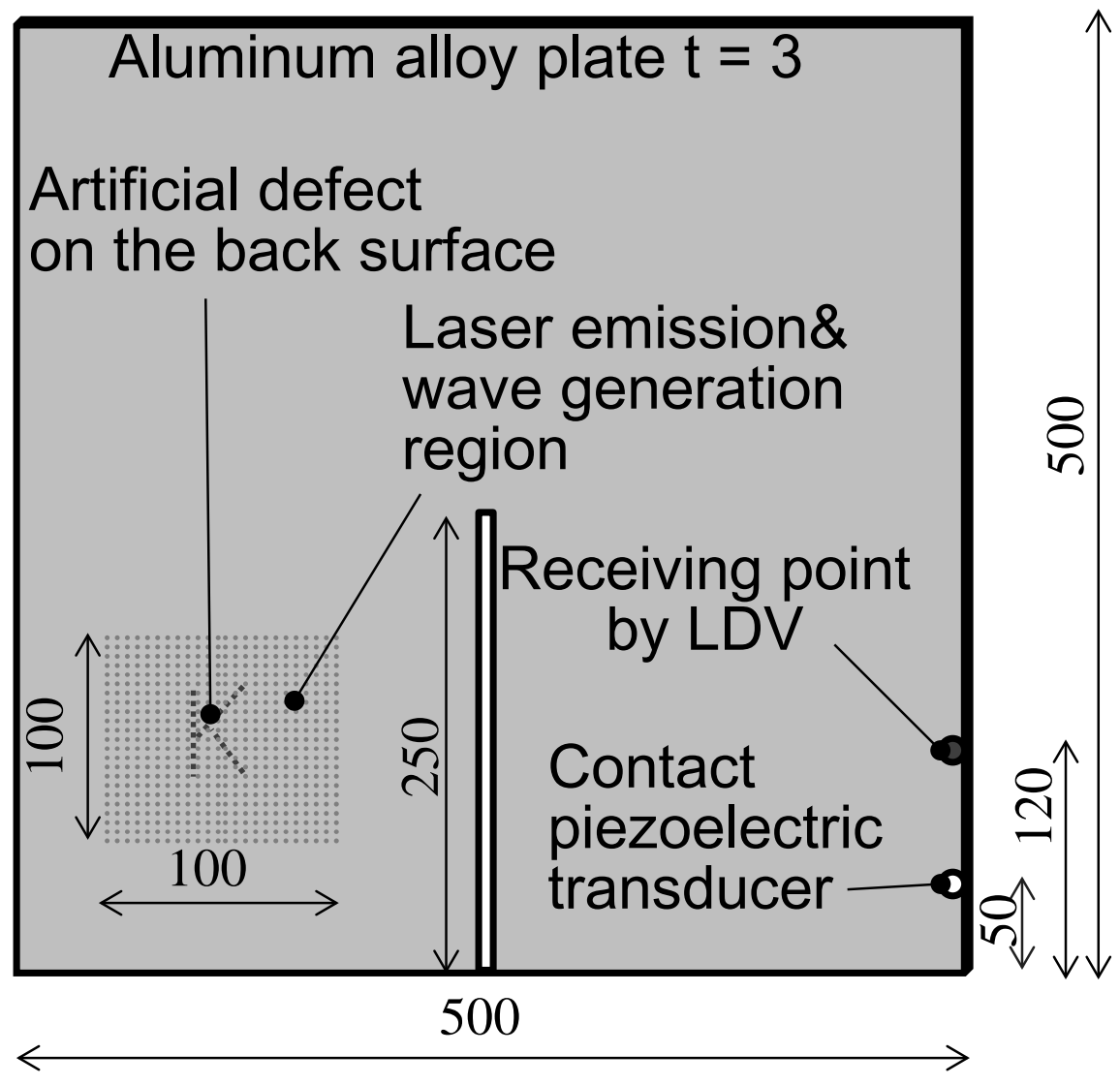


Fig. 6 Results for pulse wave generation at low scanning speed (repetition rate: $20 \mathrm{~Hz}$ ).

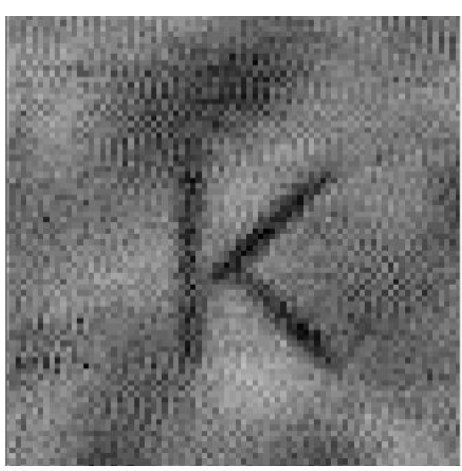

(a) RF image

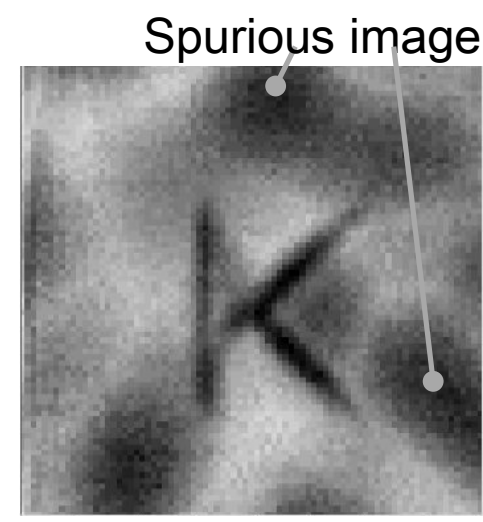

(b) FFT image

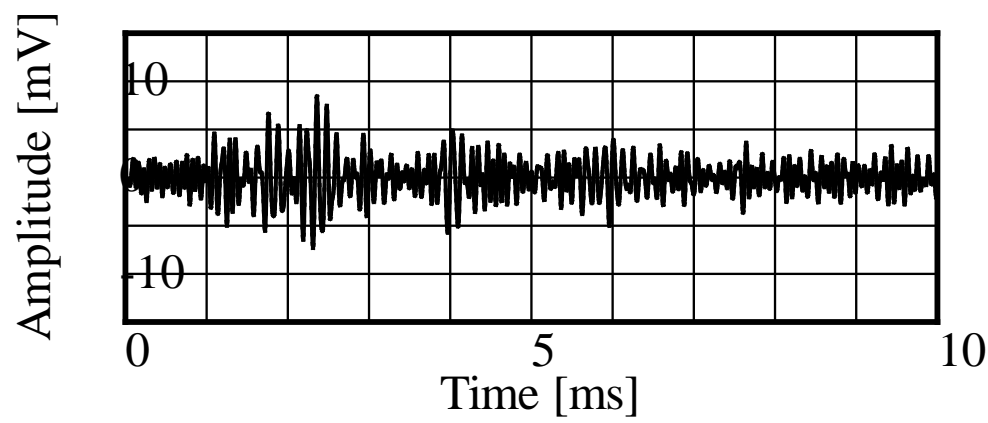

(c) Typical waveform

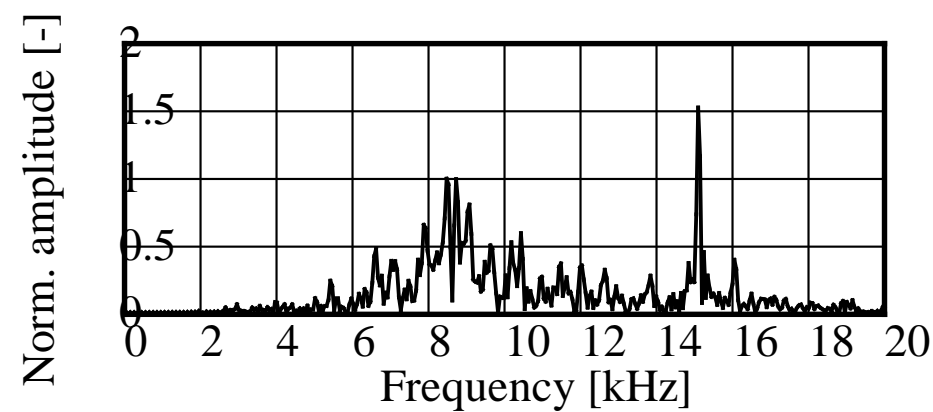

(d) Typical frequency spectrum 
Fig. 7 Results for pulse wave generation at high scanning speed (repetition rate: $200 \mathrm{~Hz}$ ).

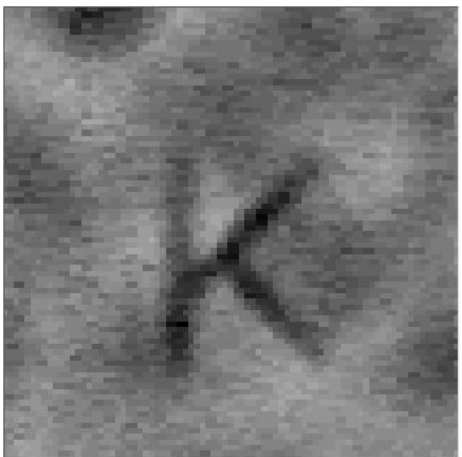

(a) RF image

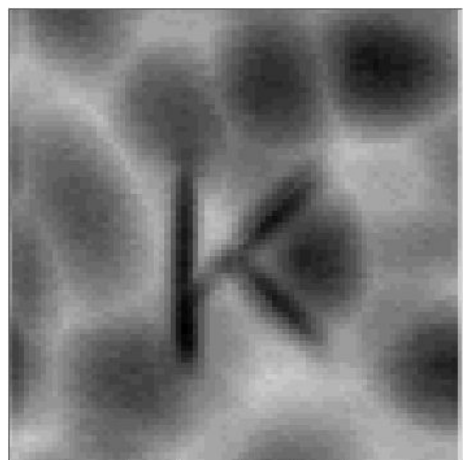

(b) FFT image

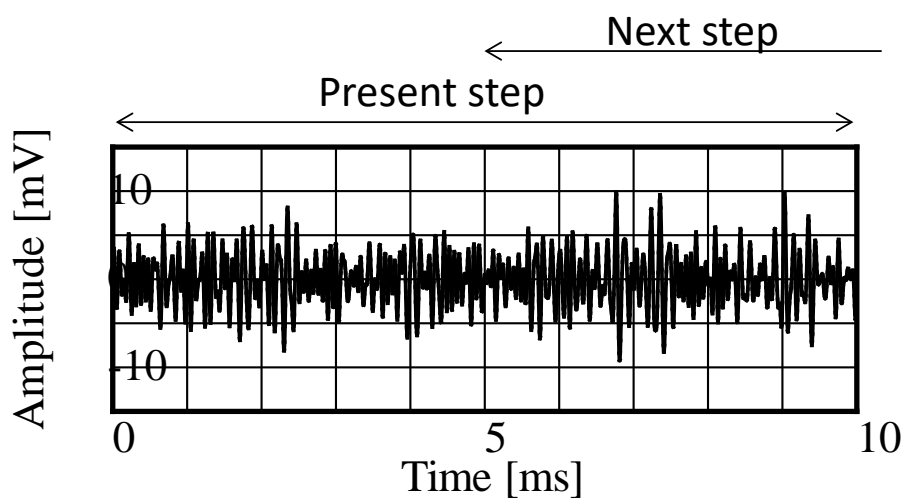

(c) Typical waveform

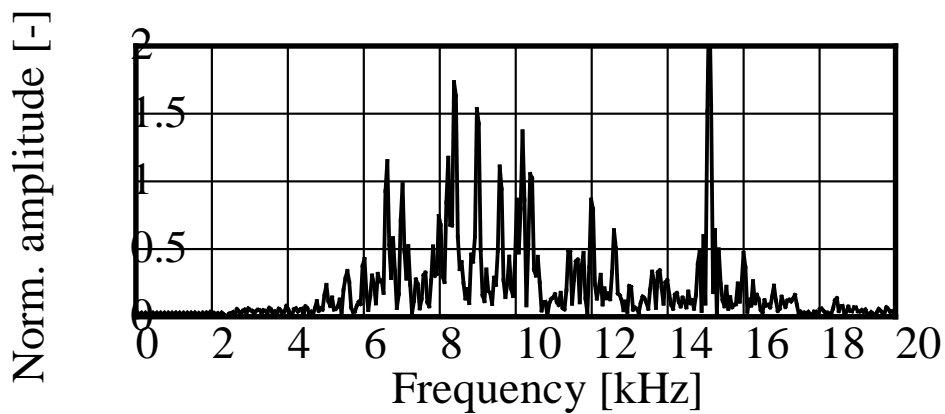

(d) Typical frequency spectrum 
Fig. 8 Images showing differences in interval of extracted waveforms. Repetition frequency: $200 \mathrm{kHz}$.

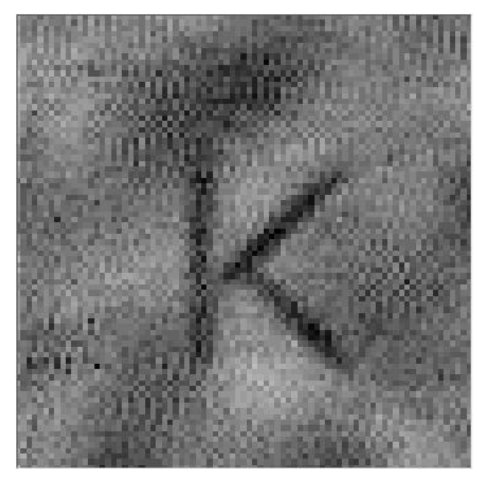

(a) RF image for $1 \mathrm{~ms}$

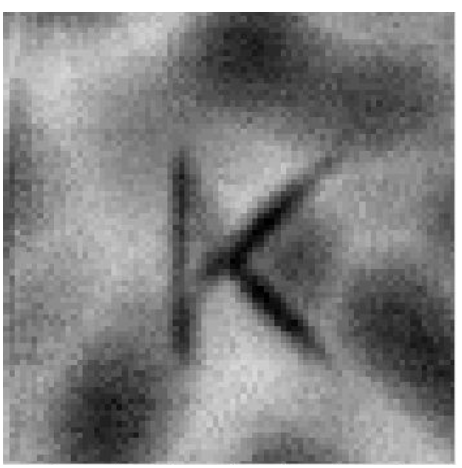

(c) FFT image for $1 \mathrm{~ms}$

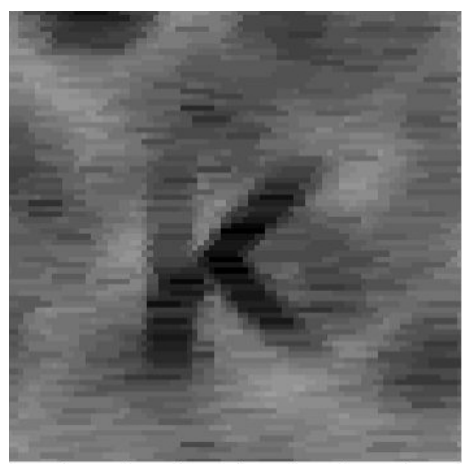

(b) RF image for $30 \mathrm{~ms}$

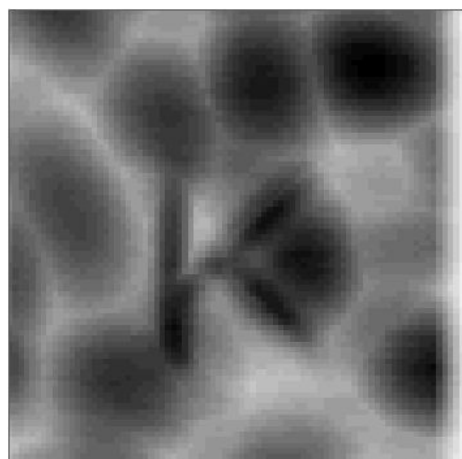

(d) FFT image for $30 \mathrm{~ms}$ 
Fig. 9 Images for different frequency bands and their averaged image. Repetition frequency: $200 \mathrm{~Hz}$. Extracted waveform interval: $10 \mathrm{~ms}$.

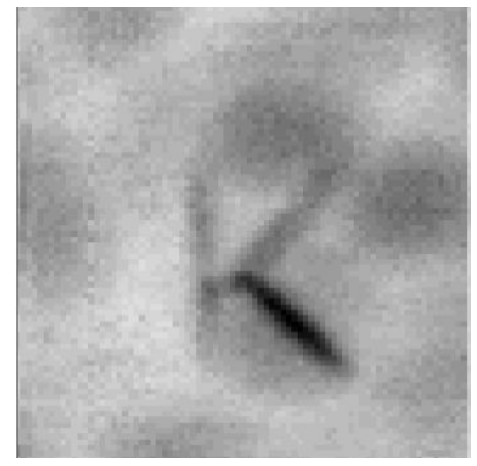

(a) $6-8 \mathrm{kHz}$

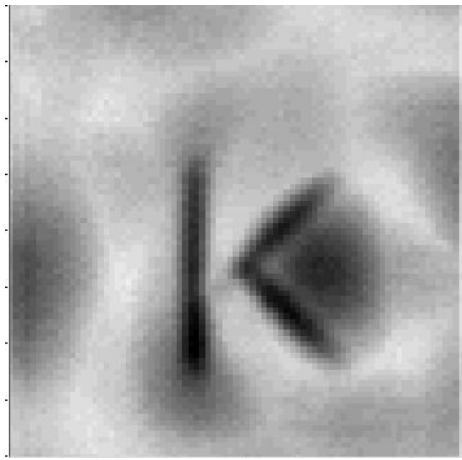

(c) $10-12 \mathrm{kHz}$

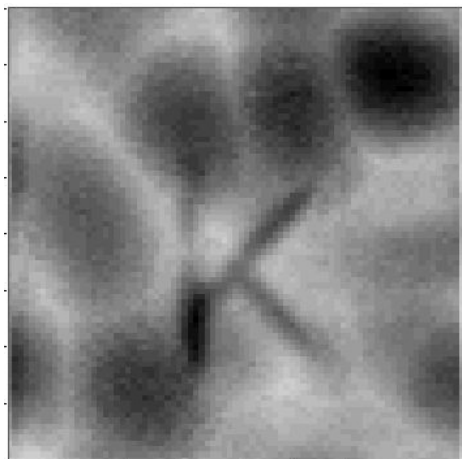

(b) $8-10 \mathrm{kHz}$

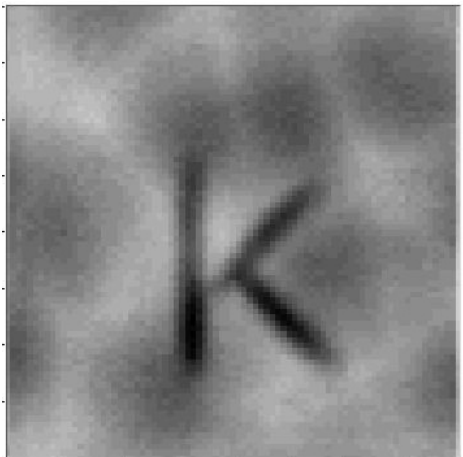

(d) Average 
Fig. 10 Frequency averaged images for high-speed scanning using broadband pulse wave.

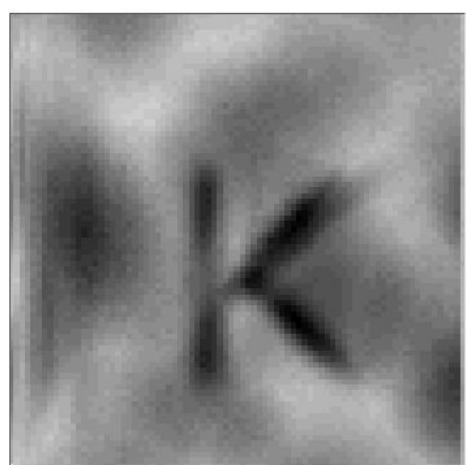

(a) $600 \mathrm{~Hz}(11 \mathrm{~s})$

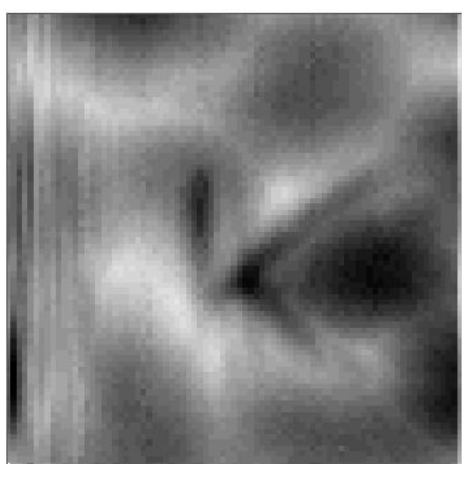

(b) $1000 \mathrm{~Hz}(6.6 \mathrm{~s})$

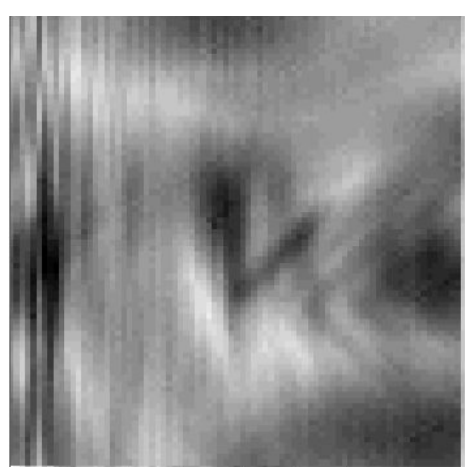

(c) $2000 \mathrm{~Hz}(3.3 \mathrm{~s})$ 
Fig. 11 Results for tone-burst wave generation at low scanning speed (repetition rate: 20 $\mathrm{Hz})$.

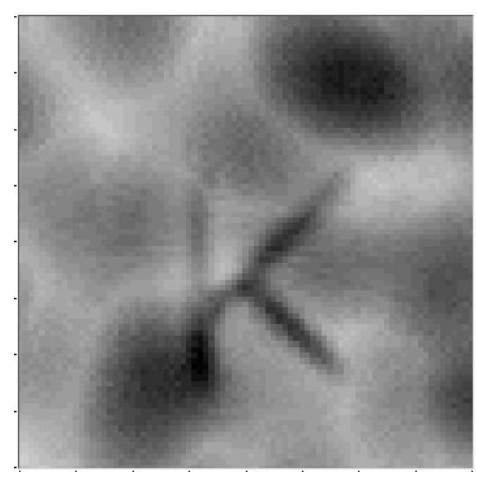

(a) RF image

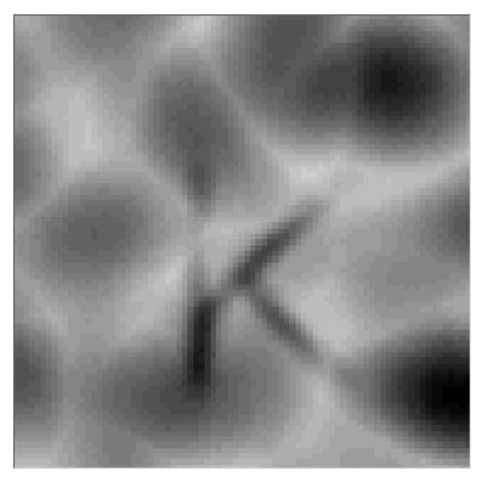

(b) FFT image

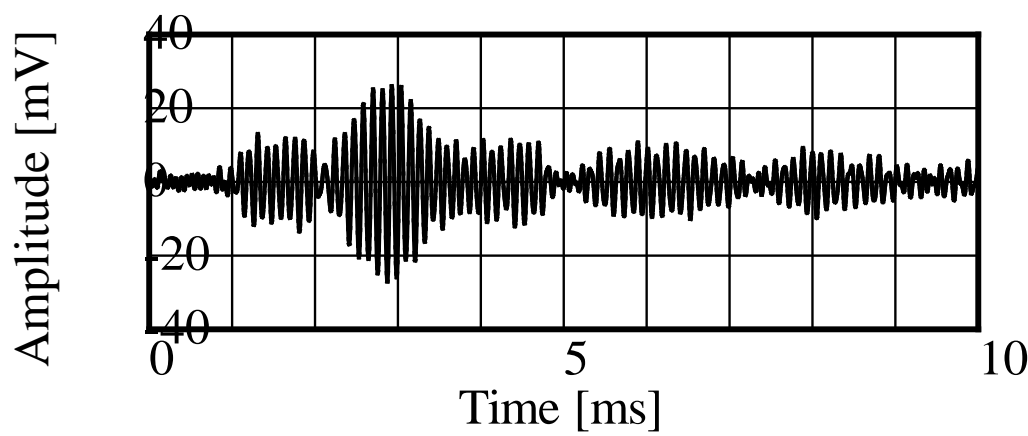

(c) Typical waveform

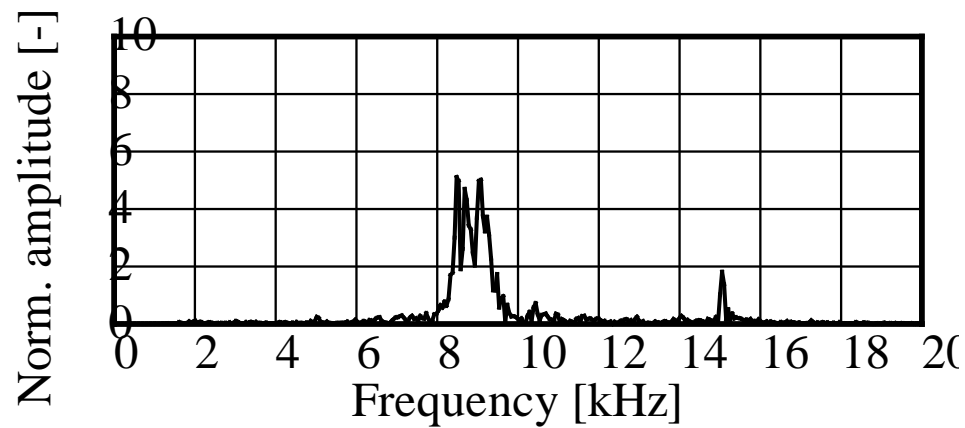

(d) Typical frequency spectrum 
Fig. 12 Results for tone-burst wave generation at high scanning speed (repetition rate: 200 $\mathrm{Hz})$.

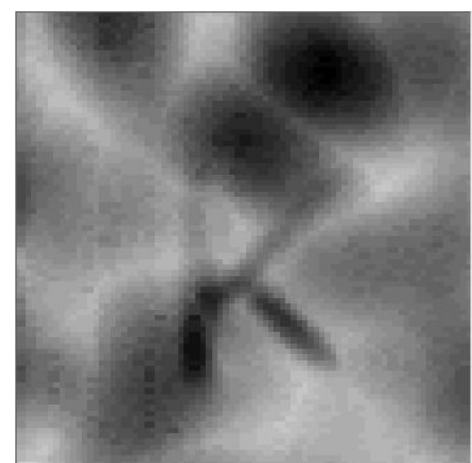

(a) RF image

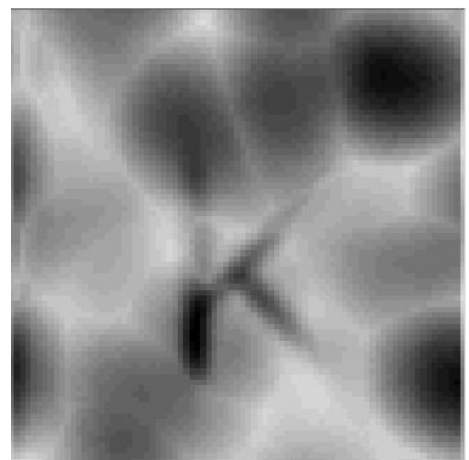

(b) FFT image

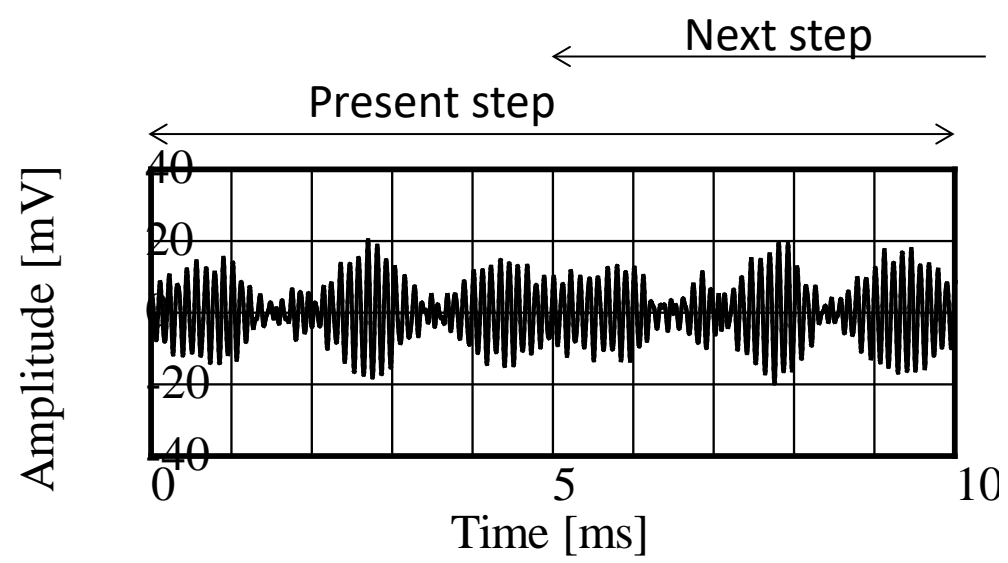

(c) Typical waveform

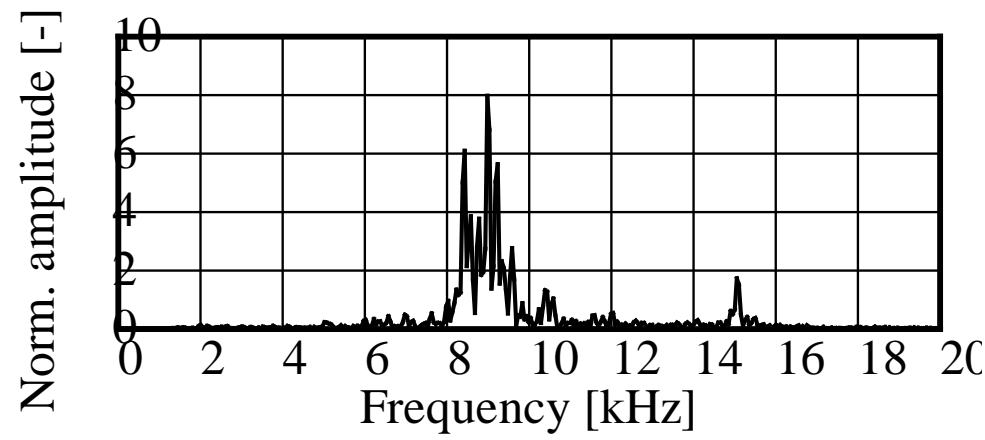

(d) Typical frequency spectrum 
Fig. 13 Images in different frequency bands, their averaged image, typical waveform, and its frequency spectrum. Multi-frequency tone burst modulation signals are used. Repetition frequency: $200 \mathrm{~Hz}$. Extract waveform interval: $10 \mathrm{~ms}$.

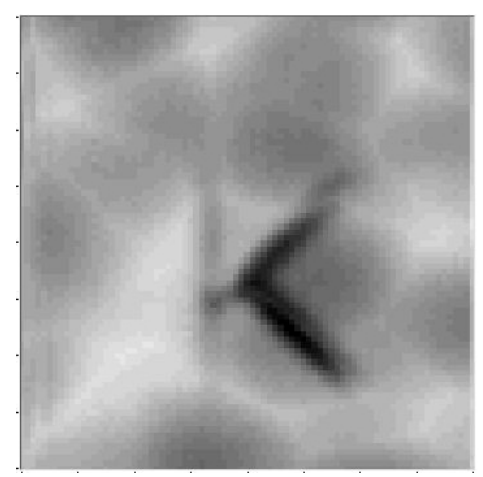

(a) $6-8 \mathrm{kHz}$

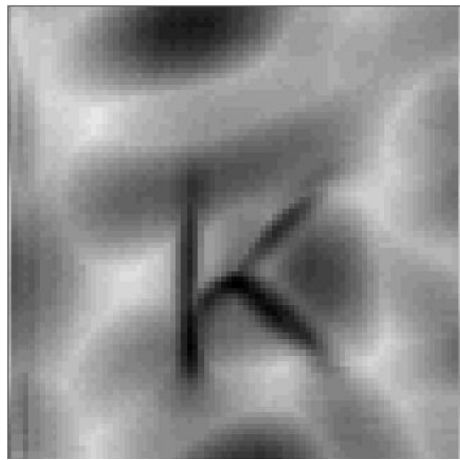

(c) $10-12 \mathrm{kHz}$

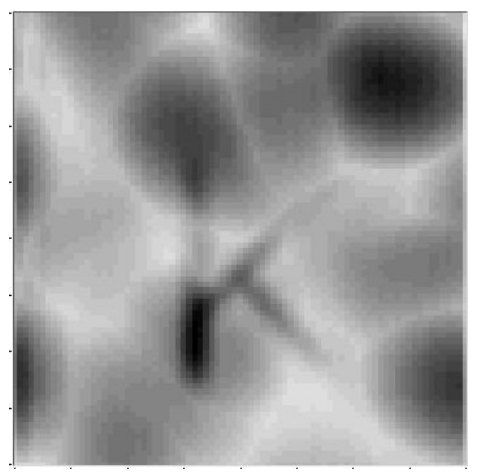

(b) $8-10 \mathrm{kHz}$

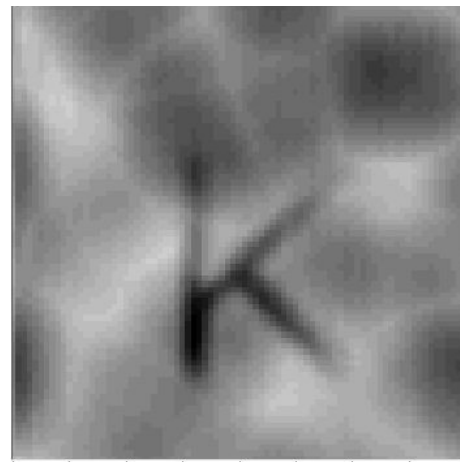

(d) Average

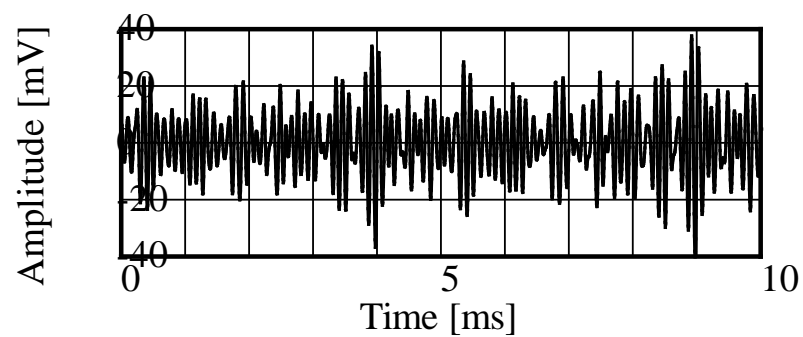

(e) Typical waveform

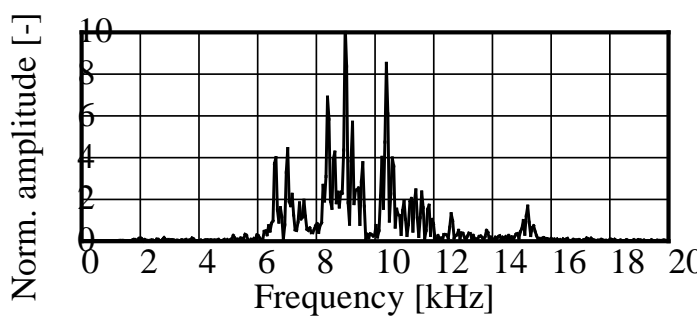

(f) Typical frequency spectrum 
Fig. 14 Frequency averaged images for high-speed scanning using multi-frequency tone-burst waves.

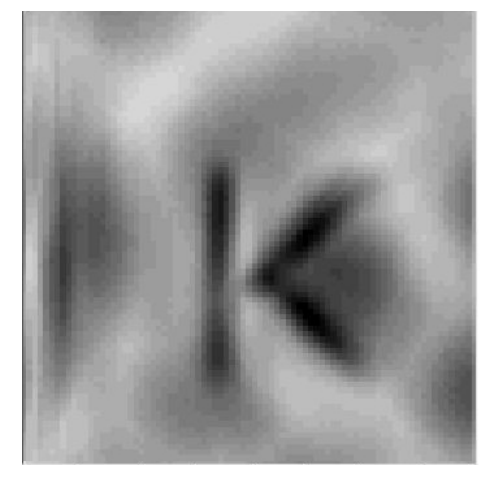

(a) $600 \mathrm{~Hz}(11 \mathrm{~s})$

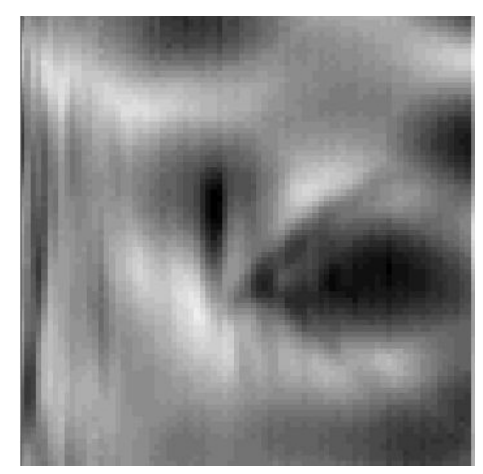

(b) $1000 \mathrm{~Hz}(6.6 \mathrm{~s})$

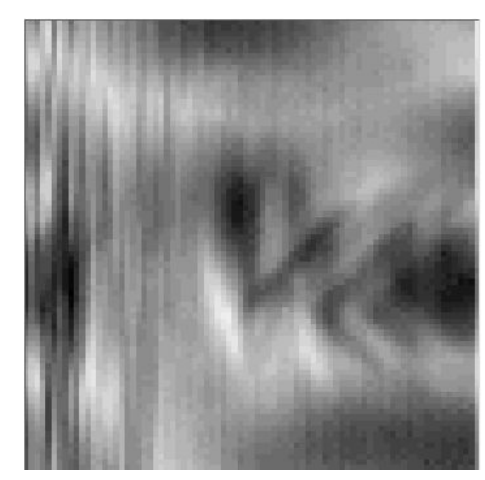

(c) $2000 \mathrm{~Hz}(3.3 \mathrm{~s})$ 
Fig. 15 Frequency averaged images obtained by measurement of LDV for pulse wave generation.

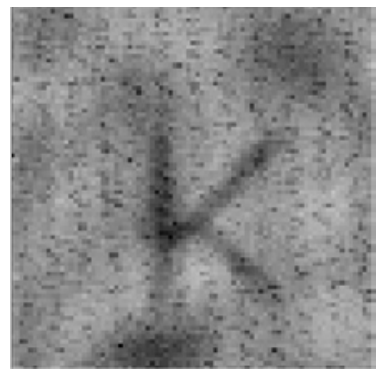

(a) $200 \mathrm{~Hz}(33 \mathrm{~s})$

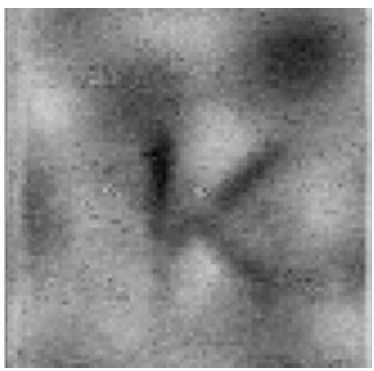

(b) $600 \mathrm{~Hz}(11 \mathrm{~s})$

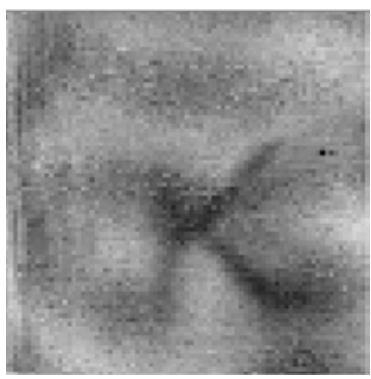

(c) $1000 \mathrm{~Hz}(6.6 \mathrm{~s})$

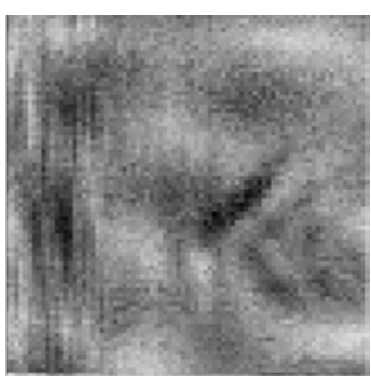

(d) $2000 \mathrm{~Hz}(3.3 \mathrm{~s})$ 
Fig. 16 Frequency averaged images obtained by measurement of LDV for multi-frequency tone-burst wave generation.

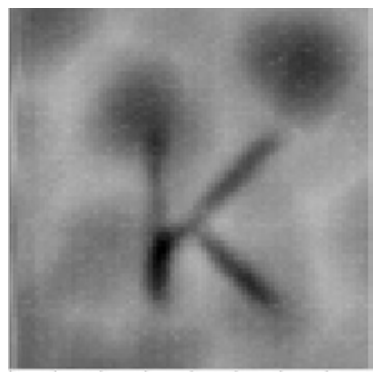

(a) $200 \mathrm{~Hz}(33 \mathrm{~s})$

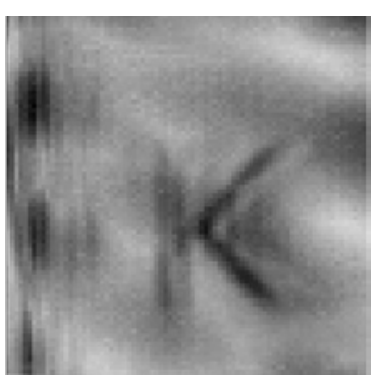

(b) $600 \mathrm{~Hz}(11 \mathrm{~s})$

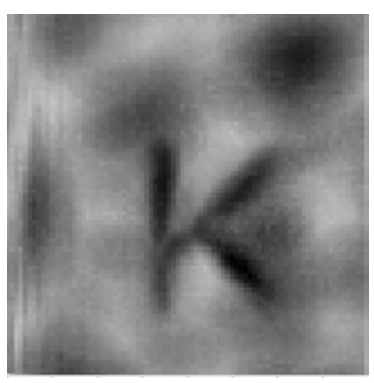

(c) $1000 \mathrm{~Hz}(6.6 \mathrm{~s})$

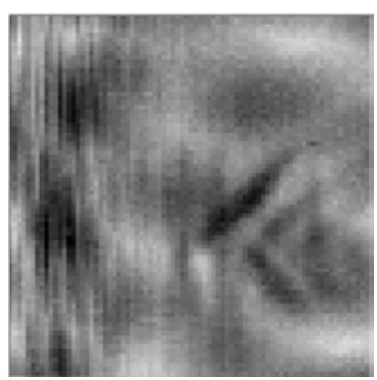

(d) $2000 \mathrm{~Hz}(3.3 \mathrm{~s})$ 